\title{
La disparition des fées dans El verdadero final de la Bella Durmiente d'Ana María Matute
}

Sylvie Ravussin

\section{(2) OpenEdition}

\section{Journals}

Édition électronique

URL : http://journals.openedition.org/edl/219

DOI : 10.4000/edl.219

ISSN : 2296-5084

\section{Éditeur}

Université de Lausanne

\section{Édition imprimée}

Date de publication : 15 décembre 2011

Pagination : $379-398$

ISBN : 978-2-940331-26-0

ISSN : 0014-2026

\section{Référence électronique}

Sylvie Ravussin, « La disparition des fées dans El verdadero final de la Bella Durmiente d'Ana María Matute », Études de lettres [En ligne], 3-4 | 2011, mis en ligne le 15 décembre 2014, consulté le 19 décembre 2020. URL : http://journals.openedition.org/edl/219; DOI : https://doi.org/10.4000/edl.219 


\section{LA DISPARITION DES FÉES DANS EL VERDADERO FINAL DE LA BELLA DURMIENTE D'ANA MARÍA MATUTE}

Cet article se propose d'étudier la façon dont Ana María Matute réécrit et adapte La Belle au bois dormant de Charles Perrault pour la jeunesse dans El verdadero final de la Bella Durmiente. L'écrivaine espagnole transforme le conte français de la fin du $\mathrm{XVII}^{\mathrm{e}}$ siècle en un cuento espagnol du $\mathrm{XX}^{\mathrm{e}}$ siècle, en recourant notamment au fantastique. Cette «reconfiguration générique» se traduit par la disparition du merveilleux et du personnage de la fée pour signaler la perte de l'enfance et les sombres réalités de l'Espagne sous le régime franquiste. Sa réécriture s'inscrit ainsi dans un projet poétique élaboré sur une longue période, dans le prolongement de Primera memoria, le roman le plus connu de Matute.

Ahora queda lejos, muy lejos,

el paisaje de la infancia, de la primera juventud.

Ana María Matute, La Trampa ${ }^{1}$

Lorsqu'elle publie El verdadero final de la Bella Durmiente ${ }^{2}$ dans la collection Grandes autores para niños (Grands auteurs pour les enfants) chez Lumen ${ }^{3}$ en 1995, Ana María Matute prend le contre-pied des

I. "Le paysage de l'enfance, de la première jeunesse, est loin, très loin maintenant", tiré de A. M. Matute, Obra completa, t. IV, p. 447, je traduis.

2. Soit La véritable fin de la Belle au bois dormant. Il s'agit d'un récit de quatre-vingtune pages, séparé en trois parties: El principe y la princesa (Le prince et la princesse), Historia de la Reina Madre y algunas cosas más (Histoire de la Reine Mère et d'autres choses encore), La madre y los niños (La mère et les enfants). Il n'existe pas de traduction française de ce texte: toutes les traductions sont de moi.

3. En 1960, Lumen avait publié l'un des premiers livres pour enfants d'Ana María Matute, El saltamontes verde, ouvrage qui débute la collection Grandes autores. Cette 
adaptations modernes de La Belle au bois dormant qui se focalisent, généralement, sur la première partie du conte français. Depuis les frères Grimm qui, les premiers, séparent le conte de Charles Perrault en deux Märchen distincts dans l'édition de 1812 des Kinder-und Hausmärchen (Dornröschen et Die Schwiegermutter), et surtout depuis le dessin animé de Walt Disney, Sleeping Beauty, en 1959, il est communément admis que cette histoire est une romance féérique qui se termine par le mariage du prince et de la princesse. Matute, quant à elle, se concentre sur la fin plus sombre du texte, qu'elle relit à partir de l'histoire récente espagnole. En effet, "tout le monde" ("todo el mundo") ${ }^{4}$ sait que la princesse endormie fut réveillée par un prince qui l'épousa, mais curieusement, "presque personne ne sait ce qui arriva ensuite» ("casi nadie sabe lo que sucedió después») ${ }^{5}$. L'écrivaine espagnole se présente ainsi comme celle qui va restituer une parole vraie, authentique, une vérité que la réception édulcorée du conte a fait oublier:

La versión que se les da a los niños se termina siempre con el beso del príncipe. Los enamorados se fueron al palacio, se casaron, fueron todos felices y comieron perdices, ¿¿no? Pero no es verdad, el cuento no termina así 6 .

Dans la seconde partie du conte de Perrault, on se souvient que la Belle est confrontée à la mère de son époux, une ogresse qui désire manger sa bru et ses petits-enfants. Grâce à la ruse du maître d'hôtel de la reine, qui échange les victimes contre des animaux, la princesse et ses deux

collection regroupe les récits adaptés pour les enfants de nombreux auteurs étrangers, tels Oscar Wilde, A. S. Pouchkine, Edgar A. Poe ou Lewis Carroll, mais aussi espagnols et contemporains d’Ana María Matute, Juan Ramón Jímenez, Ana María Moix, Carmen Martín Gaite, Esther Tusquets. Une traduction espagnole des contes de Perrault, Cuentos de hadas, a également été publiée dans cette collection en 1983. El verdadero final de la Bella Durmiente a été réédité par Lumen en 2000 dans l'anthologie Todos mis cuentos, puis de façon autonome en 2003.

4. A. M. Matute, El verdadero final de la Bella Durmiente, p. 11.

5. Ibid.

6. «La version que l'on donne aux enfants se termine toujours avec le baiser du prince. Les amoureux allèrent au palais, se marièrent, furent tous heureux et eurent beaucoup d'enfants, non? Mais ce n'est pas vrai, le conte ne se termine pas ainsi." (M.-L. Gazarian-Gautier, Ana María Matute, p. 44, je traduis). Voir aussi M. Hennard Dutheil de la Rochère, "But Marriage itself is no Party", pour un argument similaire concernant la traduction et la réécriture du conte par Angela Carter. 
enfants ont la vie sauve. Mais la reine finit par découvrir la tromperie et ordonne que les traîtres soient jetés dans une cuve pleine de serpents et de crapauds. Le retour inopiné du prince parti à la guerre permet à la famille royale d'être sauvée, la méchante reine se jetant elle-même dans la cuve.

Ana María Matute transpose cette deuxième partie du conte marquée par la persécution de la jeune épouse et de ses enfants dans le contexte socioculturel et discursif de l'Espagne de la fin du XX $\mathrm{XX}^{\mathrm{e}}$ siècle, de façon à mettre en évidence des éléments qui entrent en résonance avec l'histoire récente espagnole, et recourt pour cela au fantastique. En intitulant sa réécriture El verdadero final de la Bella Durmiente, Matute situe son récit dans la continuité du conte de Perrault, tout en signalant son écart par rapport à la convention de la fin heureuse caractéristique du genre, surtout dans les versions pour les enfants. Au début du récit, tout se passe comme si le narrateur espagnol ne faisait que reprendre le fil d'une intrigue imaginée trois siècles plus tôt par Charles Perrault. Mais cette impression est de courte durée, car Matute s'éloigne de l'univers familier pour déplacer son texte dans un autre genre en modifiant certains motifs, tels que le bois, le palais du roi et la figure de la fée.

\section{Le bois qui cachait la forêt: glissement des catégories génériques}

Le début d'El verdadero final de la Bella Durmiente raconte le voyage de noces de la Bella Durmiente et de son prince charmant, le Príncipe Azul, alors qu'ils cheminent en direction du royaume de ce dernier. Laccent est mis sur le changement et la disparition progressive du paysage connu. Les verbes utilisés renforcent cet effacement: les oiseaux «se firent progressivement de plus en plus rares» («ueron haciéndose cada vez más raros») ${ }^{7}$, les nuées de papillons "disparurent» ("desaparecieron $)^{8}$, et le bourdonnement des libellules «s'éteignit» ("se apagó») 9 . Les époux laissent «loin» («lejos») ${ }^{10}$ "derrière» («atrás») ${ }^{11}$ le monde

7. A. M. Matute, El verdadero final de la Bella Durmiente, p. 12.

8. Ibid.

9. Ibid.

Io. Ibid., p. 13.

II. Ibid., p. 12 et 16. 
familier. A leurs yeux se présente désormais une région "sombre et marécageuse» ("sombría y pantanosa») 12, peuplée d'animaux menaçants. Le couple princier pénètre alors dans un bois «différent de tous ceux qu'on connaît" ("diferente a todos los conocidos») ${ }^{13}$ : «Era un bosque salvaje, obstruido por raíces gigantescas, donde abrirse camino requería gran esfuerzo " ${ }^{14}$ ("C'était un bois sauvage, obstrué par des racines gigantesques, où se frayer un chemin requérait un grand effort»). Ce bois rappelle bien sûr celui de La Belle au bois dormant, qu'une fée prend soin de faire pousser autour du château pour protéger la princesse des curieux. Comme chez Matute, le bois du conte français est difficile à franchir: les arbres et les ronces sont si entrelacés que «beste ny homme n'y auroit pû passer " ${ }^{15}$. Mais chez Perrault, le prince n'a pas besoin de s'ouvrir un passage dans la végétation car "A peine s'avança-t-il vers le bois, que tous ces grands arbres, ces ronces $\&$ ces épines s'écarterent d'elles-mesmes pour le laisser passer » ${ }^{16}$. A l'inverse, le passage n'est plus facilité par une intervention merveilleuse pour la Bella Durmiente et le Príncipe Azul. En reprenant et en modifiant le motif du bois impénétrable, Matute indique que, si sa réécriture s'inscrit dans la continuité de La Belle au bois dormant, elle s'en éloigne également. Son bois n'est ni féérique, ni magique, mais «ensorcelé" ("embrujado») ${ }^{17}$. Ainsi, plus les personnages avancent dans leur voyage, plus le monde connu et merveilleux s'efface autour d'eux, cédant la place à un autre univers inquiétant. Selon moi, ces longues descriptions extrêmement détaillées et placées en début de récit ont une autre fonction que la simple narration du voyage de retour dans le royaume du prince. En insistant sur la disparition du merveilleux, le narrateur invite le lecteur à s'interroger sur ce qu'il est en train de lire et à questionner ses attentes génériques. Car lui aussi, au fil des pages, s'apprête à entrer en territoire inconnu, loin des contes familiers peuplés de fées. L'éloignement géographique des personnages et la transformation du paysage correspondent donc à un éloignement générique ${ }^{18}$. Alors que

I2. Ibid., p. 12.

I3. Ibid., p. 13.

I4. Ibid.

15. Ch. Perrault, «La Belle au bois dormant», p. 18.

I6. Ibid., p. 22.

17. A. M. Matute, El verdadero final de la Bella Durmiente, p. 13.

I8. Matute a souvent fait part de son indignation envers les versions tronquées, infantilisées ou politiquement correctes des contes, comme celles largement diffusées 
le titre de la réécriture et le premier paragraphe du texte font explicitement référence à des éléments clés de l'intrigue - l'endormissement de la princesse, son réveil et son mariage avec le prince -, les motifs que le lecteur rencontre par la suite se démarquent de cette vision stéréotypée et réorientent le conte vers le fantastique ${ }^{19}$.

Dans son Introduction à la littérature fantastique, Tzvetan Todorov distingue quatre catégories génériques très proches: l'étrange pur, le fantastique-étrange, le fantastique-merveilleux et le merveilleux pur. Dans ce dernier cas, écrit-il, "les éléments surnaturels ne provoquent aucune réaction particulière ni chez les personnages, ni chez le lecteur implicite ${ }^{20}$. Il en va ainsi du conte de Perrault, car la présence de fées «ne surprend pas dans le monde du Il était une fois» ("no sorprende en el mundo del Erase o había una vez») ${ }^{21}$. Les personnages de La Belle au bois dormant ne sont pas étonnés que celles-ci prodiguent des dons à une princesse comme "c'estoit la coustume des Fées en ce temps là»22. De la même manière, le fait qu'une fée endorme de sa baguette tous les habitants d'un palais n'est pas déconcertant. Mais si personne ne s'interroge sur la présence du merveilleux dans le conte français, ce n'est plus le cas dans la réécriture de Matute: la Bella Durmiente est en effet consciente des changements qui se produisent autour d'elle, comme l'indiquent les nombreuses questions qu'elle adresse au Príncipe Azul au début du récit. Tout d'abord, durant son voyage de noces, la princesse distingue des créatures dans les herbes hautes, mais «elle n'arrivait pas à dire si elles étaient vraies ou si elle les avait imaginées ou confondues avec des insectes, des petits animaux ou de minuscules créatures du

par les dessins animés de Disney, qu’elle qualifie de «manipulation» («manipulación», J. M. de Prada, "Yo no soy una erudita, ni falta me hace», p. 18, je traduis). Elle s'inscrit contre la "Disneyfication» du conte, à l'instar d'auteures contemporaines comme Angela Carter et Jane Yolen : voir à ce propos M. Hennard Dutheil de la Rochère et G. Viret dans ce volume.

19. Dès son apparition en Espagne au début du XIX ${ }^{\mathrm{e}}$ siècle, le genre connut un franc succès. Les premiers textes d'auteurs espagnols, publiés d'abord dans des revues, datent des années 1830. Au sujet de l'histoire des genres fantastique et gothique en Espagne, voir D. Roas, De la maravilla al horror.

20. T. Todorov, Introduction à la littérature fantastique, p. 59. Voir aussi dans ce volume l'article de C. François, qui a aussi recours aux catégories de Todorov pour distinguer les contes de Perrault et les Märchen apparentés des Grimm.

2I. J.-M. Adam, C. U. Lorda, Lingüistica de los textos narrativos, p. 140, je traduis.

22. Ch. Perrault, La Belle au bois dormant, p. 3. 
fond des fourrés" ("ella no sabía decirse si fueron verdaderas o las había imaginado o confundido con insectos, pequeños animales o diminutas criaturas del fondo de la maleza») ${ }^{23}$. Dans ce bois, la réalité échappe à la princesse: elle est incertaine quant à la nature de ce qu'elle voit, ou croit voir. Le glissement dans un autre genre s'amorce dans cette transformation de la forêt merveilleuse en un univers sombre, grouillant et minuscule qui trouvera son point culminant dans l'arrivée au château de la mère du prince. Il est dès lors particulièrement significatif que la belle-mère de la Bella Durmiente s'appelle la reine Selva, terme qui signifie "forêt", comme si elle incarnait en quelque sorte ce monde obscur et inquiétant qu'est le mystérieux bois dormant du conte classique, et qui fait basculer le récit dans le fantastique. Plus loin, alors qu'elle observe la reine mère attraper un oiseau dans son jardin, la princesse remarque que:

El ave desaparecía entre las manos de la Reine Madre, como si se esfumara. La Princesa supuso que lo guardaría en alguna jaula, porque no los mataba, ni los ordenaba matar. Pero nunca vio jaula alguna, ni pájaro, grande o pequeño, por parte alguna del castillo ${ }^{24}$.

Dans le premier cas, la Bella Durmiente se demande si elle a réellement vu des créatures; dans le second, elle est sûre que l'oiseau s'est volatilisé, mais ne sait pas comment interpréter cette disparition: sa perception du monde devient incertaine. Cela correspond à la définition du fantastique proposée par Todorov, comme la «ligne de partage entre l'étrange et le merveilleux ${ }^{25}$. Autrement dit, le personnage est confronté à un événement qui peut être expliqué par des causes naturelles ou par des causes surnaturelles, et cette hésitation "crée l'effet fantastique " ${ }^{26}$. Le château de la reine Selva sert de pivot entre ces deux genres:

Sobre un montículo rocoso, rodeado de niebla, apareció la silueta de un castillo. Parecía formar parte de la niebla, era en sí mismo como

23. A. M. Matute, El verdadero final de la Bella Durmiente, p. 13.

24. "L'oiseau disparaissait entre les mains de la Reine Mère, comme s'il se volatilisait. La Princesse supposa qu'elle le gardait dans une cage, parce qu'elle ne les tuait pas, ni n'ordonnait qu'on les tue. Mais elle ne vit jamais aucune cage, ni oiseau, grand ou petit, dans aucune partie du château.» (ibid., p. 19).

25. T. Todorov, Introduction à la littérature fantastique, p. 31.

26. Ibid., p. 30. 
una figura hecha de niebla aún más oscura, de contornos imprecisos. [...] Cuando ya se hallaban frente al castillo, la Bella Durmiente pudo ver que de su foso surgía una especie de neblina muy oscura, y que un olor a fango y raíces podridas brotaba de él, mezclándose al chapoteo de animales que ella no conocía ${ }^{27}$.

Le château perché sur un rocher et entouré de brouillard est fortement connoté. Loin du palais merveilleux tel qu'il est décrit dans le conte français, il se rapproche des châteaux que l'on trouve dans les Gothic novels et les récits de vampires. Alors que chez Perrault, le prince allait chercher sa femme "en grande cérémonie ${ }^{28}$ et lui faisait "une entrée magnifique dans la Ville Capitale " ${ }^{29}$, dans le cuento, la Bella Durmiente est plongée dès son arrivée dans une atmosphère froide, grise et lugubre. C'est dans ce lieu inquiétant qu'elle rencontre pour la première fois sa belle-mère, qui ressemble à un vampire (fig. 1) ${ }^{30}$. La reine mère donne une impression d'éternelle jeunesse, avec sa peau claire et «si fine que les veines transparaissaient» («tan fina que transparentaban las venas») ${ }^{31}$, et un visage «mince et presque sans rides, très pâle, couronné par des cheveux [...] aussi noirs que pourrait les avoir une fille de vingt ans" ("delgado y apenas sin arrugas, muy pálido, coronado por cabellos [...] tan negros como los podría tener una muchacha de veinte años») ${ }^{32}$. Le texte mentionne

27. «Sur un monticule rocheux, entouré de brouillard, apparut la silhouette d'un château. Il semblait faire partie du brouillard, il était lui-même comme une figure faite de brouillard encore plus obscur, aux contours imprécis. [...] Alors qu'ils se trouvaient en face du château, la Belle au bois dormant put voir que de son fossé surgissait une espèce de brouillard très obscur, et qu'une odeur de boue et de racines pourries en jaillissait, se mélangeant au clapotis d'animaux qu'elle ne connaissait pas.» (A. M. Matute, El verdadero final de la Bella Durmiente, p. 16).

28. Ch. Perrault, La Belle au bois dormant, p. 33.

29. Ibid.

30. Cette impression est renforcée par les illustrations en noir et blanc de Teresa Ramos, qui la représente systématiquement la bouche ouverte et les canines saillantes. Concernant l'hybridation du conte de fée et de la tradition gothique anglaise, voir l'article, dans ce volume, de M. Hennard Dutheil de la Rochère, que je remercie pour ses suggestions et ses relectures attentives de mon article. Elle montre comment, dans la pièce radiophonique Vampirella et la nouvelle qui s'en inspire, The Lady of the House of Love, Angela Carter revisite le mythe de la «femme fatale» en fusionnant la figure de la Belle et sa belle-mère ogresse.

3I. A. M. Matute, El verdadero final de la Bella Durmiente, p. 18.

32. Ibid. 
Fig. 1 - Plus les personnages avancent dans leur voyage, plus le monde connu et merveilleux s'efface autour d'eux, cédant la place à un univers sombre et inquiétant. 
également que "le soleil n'[...] entrait jamais» ("el sol no entraba nunca [...] $)^{33}$ dans ses pupilles. Enfin, le sourire de Selva découvre deux longues canines blanches, qui deviennent parfois «rouges comme le sang" («rojos como la sangre») ${ }^{34}$. La reine, véritable prédatrice, est née avec toutes ses dents et, petite, se nourrissait déjà de viande crue.

Pour Matute, la reine mère est «le personnage essentiel» ("el personaje esencial» ${ }^{35}$ du texte: c'est sur elle que se concentre le second chapitre de sa réécriture. Cette partie, qui met en lumière le passé de la mère du Príncipe Azul, est sa principale innovation par rapport au conte de Perrault. Selva n'appartient donc pas au monde du conte merveilleux, comme les créatures qui l'accompagnent partout et que la Bella Durmiente imagine appartenir "à un autre monde ténébreux» ("a otro mundo tenebroso") ${ }^{36}$. Matute n'est pas la seule auteure espagnole de la fin du $\mathrm{XX}^{\mathrm{e}}$ siècle à recourir au genre néo-gothique ${ }^{37}$. Dans son article "Contemporary Spanish Women Writers and the Feminine NeoGothic", Janet Pérez explique la mobilisation de ce genre par les écrivaines espagnoles au $\mathrm{XX}^{\mathrm{e}}$ siècle en réponse au contexte dictatorial du régime franquiste:

The original flowering of gothic coincided with massive social reorganization - whether from industrialization, political revolution, and democratization, or some combination of these - together with breakdowns in traditional class boundaries, as happens with the emergence of the neo-gothic mode in late twentieth-century Spain. [...] The postindustrial, postrevolutionary context in which gothic emerges - and neo-gothic later - is a world changed forever and, in significant ways, apocalyptic. Post-Franco literature - of which neogothic is part - reflects such apocalyptic change ${ }^{38}$.

33. Ibid.

34. Ibid., p. 27.

35. M.-L. Gazarian-Gautier, Ana Maria Matute, p. 43, je traduis.

36. A. M. Matute, El verdadero final de la Bella Durmiente, p. 55.

37. Citons par exemple Cristina Fernández Cubas (El columpio, 1995), Adelaida García Morales (El silencio de las sierras, 1985; La lógica del vampire, 1990; La tía Agueda, 1995; La señorita Medina, 1997) ou encore Marina Mayoral (Dar la vida y el alma, 1996).

38. J. Pérez, "Contemporary Spanish Women Writers and the Feminine NeoGothic», p. 126. 
L'impact du recours au fantastique dans El verdadero final de la Bella Durmiente, qui s'inscrit dans cette mouvance, est encore accentué par la disparition de tous les éléments merveilleux, comme je vais le montrer dans ce qui suit.

\section{La disparition des fées et la perte de l'enfance merveilleuse}

Personnage merveilleux par excellence, la fée occupe une place prépondérante chez Perrault. Sept fées sont invitées au baptême de la princesse et chacune lui fait un don, outre la méchante fée. Il est particulièrement significatif, dans El verdadero final de la Bella Durmiente, que les époux princiers, en se remémorant la malédiction lancée par une fée "perverse» ("perversa») ${ }^{39}$ sur la Bella Durmiente, décident de baptiser leur fille Aurora "dans la plus stricte intimité, sans invitations à des fées ni rien de semblable» (« en la más estricta intimidad, sin invitaciones a hadas ni cosa parecida" ${ }^{40}$. Ils ne connaissent de toute façon "aucune fée ni personne qui y ressemblât» ("a ninguna hada ni a nadie que se le pareciera " ${ }^{41}$. Chez Matute, les fées ne président donc plus au destin du nouveau-né. Quant à la jeune fée "grandement prévoyante» ${ }^{42}$, qui réduit la condamnation à mort en un sommeil de cent ans et endort le château du roi chez Perrault, elle est remplacée par les parents de la Bella Durmiente. Il est ainsi indiqué que si des veneurs, des rabatteurs et d'autres habitants du château accompagnent la princesse après son réveil, c'est "grâce à ses parents qui avaient été prévoyants" ("gracias a lo previsores que habían sido sus padres") ${ }^{43}$. Remarquons enfin que le seul personnage pratiquant la magie dans le cuento est Floresta, la mère de Selva, qui redonne vie au royaume ruiné du roi Abundio grâce à ses pouvoirs. Son prénom, signifiant "bosquet", est associé à une nature riante et joyeuse, qui contraste avec la personnalité sombre et mystérieuse de Selva. Mais, malgré ses actions féeriques, Floresta est qualifiée

39. A. M. Matute, El verdadero final de la Bella Durmiente, p. 12.

40. Ibid., p. 20.

4I. Ibid.

42. Ch. Perrault, La Belle au bois dormant, p. 15.

43. A. M. Matute, El verdadero final de la Bella Durmiente, p. 12. 
de «sorcière» ${ }^{44}$ («bruja $)^{45}$ pratiquant la magie blanche. Si les fées sont évoquées dans El verdadero final de la Bella Durmiente, c'est pour souligner leur absence ou leur inaccessibilité, à l'image de «la cascade de la Source des Fées» («la cascada del Manantial de las Hadas») ${ }^{46}$, que la princesse et ses enfants observent, cachés dans les combles de la maison de campagne de Selva, à travers le trou d'un volet, qui "paraissait être si près d'eux, et, cependant, si loin" ("parecía estar tan cerca de ellos, $y$, sin embargo, tan lejos $»)^{47}$. Il en est de même pour tous les autres éléments liés au merveilleux. Les termes «merveille» («maravilla») et «merveilleux» («maravilloso») apparaissent toujours dans des passages en lien avec le passé ou l'enfance lointaine de la princesse. Le merveilleux se rapporte à des événements et des périodes révolus, et toujours en relation avec un questionnement ou une inquiétude concernant la situation présente. Durant le voyage de retour, la Bella Durmiente demande au prince:

- Cuando me despertaste con un beso, los árboles y los arbustos florecían, y la hierba, y hasta las ortigas, despedían un maravilloso perfume, que nunca olvidaré... ¿Qué ha pasado? ¿Por qué han desaparecido el canto de los mirlos, y las flores, y el sol ? ${ }^{48}$

Tout au long du texte, la princesse s'accroche à ses souvenirs d'enfance. Lorsqu'elle se retrouve seule entre les mains de sa belle-mère après le départ du Príncipe Azul à la guerre, plusieurs éléments en lien avec son enfance la préviennent du danger qu'elle court: le lierre qui recouvre la fenêtre de sa chambre, arrière-arrière-arrière petite-fille du lierre qui ornait la muraille du château de son père, mais aussi les oiseaux, dont sa nourrice, «de plus en plus regrettée» ("cada vez más añorada») ${ }^{49}$, lui avait appris le langage. L'évocation de ces éléments permet d'établir

44. Ce terme, évoquant un autre chemin générique que celui du conte merveilleux, est déjà présent chez Perrault.

45. A. M. Matute, El verdadero final de la Bella Durmiente, p. 44.

46. Ibid., p. 71.

47. Ibid.

48. «- Lorsque tu m'as réveillée avec un baiser, les arbres et les arbustes fleurissaient, et l'herbe, et même les orties, répandaient un merveilleux parfum, que jamais je n'oublierai... Que s'est-il passé? Pourquoi le chant des merles, et les fleurs, et le soleil ont-ils disparu?» (ibid., p. 14, je souligne).

49. Ibid., p. 17. 
un contraste entre un avant merveilleux et un présent désenchanté et inquiétant, la nature bienveillante et la douce nourrice étant le pendant positif de la méchante reine Selva. L'éloignement géographique du début $\mathrm{du}$ texte se transforme ici en un éloignement temporel. L'insistance sur ce qui reste "loin" et "derrière" durant le voyage de noces du prince et de la princesse peut ainsi être interprétée comme l'abandon de l'enfance et le passage à l'âge adulte. La Bella Durmiente est en effet le seul personnage de la réécriture à avoir connu, cent ans auparavant, le monde du conte et à devoir affronter cette nouvelle réalité. Les autres habitants du château, réveillés en même temps qu'elle, se sont enfuis durant le voyage, car ils n'étaient pas adaptés à la cruauté du monde tel qu'il est devenu. La princesse, qui n'a pas encore atteint l'«âge de raison" ("edad de la razón $)^{50}$, se retrouve face à une réalité qu'elle "n'avait pas eu l'occasion de vivre" ("no había tenido oportunidad de vivir») ${ }^{51}$. Ana María Matute relègue ainsi le merveilleux du conte dans le passé et montre la confrontation de la princesse, à peine réveillée, avec le monde adulte. Désormais, celui-ci n’est plus enchanté mais "méchant et inconnu»:

Llena de zozobra y desánimo, regresó a su habitación y, por algunos instantes, pensó que, si no fuera por el recuerdo del Príncipe Azul y la felicidad que con él, hasta el momento, había vivido, hubiera deseado no despertar de su largo sueño para encontrarse en un mundo tan feroz, malvado y desconocido ${ }^{52}$.

On peut lire dans cette nostalgie de l'enfance, associée à une nature enchantée et aux récits de sa nourrice ${ }^{53}$, une réflexion sur le conte de fées, genre aujourd'hui révolu (en tout cas dans sa forme Disneyfiée).

5o. Ibid., p. 16.

5I. Ibid., p. 26.

52. "Pleine d'angoisse et de découragement, elle retourna dans sa chambre et, pendant quelques instants, elle pensa que, si ce n'était pour le souvenir du Prince Charmant et le bonheur qu'avec lui, jusqu'à maintenant, elle avait vécu, elle aurait désiré ne pas se réveiller de son long sommeil pour se retrouver dans un monde aussi féroce, méchant et inconnu.» (ibid., p. 56). Dans ce volume, M. Viegnes montre que le thème du sommeil de la Belle est très présent chez les auteurs français du XIX ${ }^{\mathrm{e}}$ siècle. Il cite notamment le récit de La Belle au bois rêvant de Catulle Mendès, dans lequel la princesse préfère rester endormie et vivre dans le monde des rêves que dans celui de la réalité.

53. La figure de la nourrice est associée chez Perrault au genre du conte, notamment dans la préface du recueil Griselidis, nouvelle, avec le conte de Peau d'Asne et celuy des Souhaits ridicules (1694), aussi appelé Contes en vers. 
La Bella Durmiente tente d'interpréter sa nouvelle réalité à la lumière de ce qu'elle a appris durant son enfance au château de ses parents. Ainsi remarque-t-elle que le bois obscur qu'elle traverse «ne ressemblait en rien aux bois [qu'elle] se rappelait de son enfance» ("No se parecía en nada a los bosques [que ella] recordaba de su niñez») ${ }^{54}$ ou que les créatures inconnues qu'elle voit ne lui avaient été mentionnées que par sa nourrice "durant son enfance» ("en su infancia») 55 . Le recours au fantastique permet de montrer l'inadéquation entre ce que la princesse connaît et ce qu'elle voit mais ne sait pas interpréter. El verdadero final de la Bella Durmiente met ainsi en scène ce que Margaret Jones décrit comme "un choc brutal avec la réalité» ("a sharp clash with reality») ${ }^{56}$. Ce choc marque une nette séparation entre le monde des enfants et celui des adultes, que Matute considère comme «des mondes complètement différents» ("mundos completamente diferentes») ${ }^{57}$. Cette cassure entre deux âges de la vie, que Matute dit avoir elle-même ressentie au début de la guerre civile espagnole alors qu'elle avait dix ans ${ }^{58}$, correspond à une rupture historique. Elle est présente dans plusieurs de ses livres ${ }^{59}$, et est mentionnée dans la biographie qui précède le cuento dans l'édition de 1995. Il y est écrit que cet événement «changea totalement sa vie, bouleversa son petit monde» ("cambió totalmente su vida, trastornó su pequeño mundo") ${ }^{60}$. La lecture psychologique d'El verdadero final de la Bella Durmiente se double donc d'une lecture politique. Réécrire la seconde partie de La Belle au bois dormant permet ainsi à Matute de mettre en scène cette rupture à la fois personnelle et collective, pour mieux dire la cruauté du monde adulte et de la dictature, d'où il n'y a pas d'échappatoire possible. C'est cela qu'exprime le dernier paragraphe de son cuento:

Pero debe suponerse que, tal y como suelen terminar estas historias, fueron todos muy felices. Aunque la Princesa nunca más sería

54. A. M. Matute, El verdadero final de la Bella Durmiente, p. 13.

55. Ibid.

56. M. E. W. Jones, The Literary World of Ana Maria Matute, p. 58, je traduis.

57. M.-L. Gazarian-Gautier, Ana María Matute, p. 32, je traduis.

58. Voir notamment M.-L. Gazarian-Gautier, Ana María Matute, p. 72.

59. Notamment El saltamontes verde (1960), El aprendiz (1960), Carnivalito (1962), Soló un pie descalzo (1983), Primera memoria (1960), Los niños tontos (1956), El Arrepentimiento y otras narraciones (1961) ou encore Algunos muchachos (1964).

6o. A. M. Matute, El verdadero final de la Bella Durmiente, p. 7. 
tan cándida, ni el Príncipe tan Azul, ni los niños tan ignorantes e indefensos ${ }^{61}$.

La conjonction concessive "aunque» renverse le traditionnel happy end des histoires de princes et de princesses. Avec cette dernière remarque, le narrateur se montre très critique à l'égard des personnages. La "reconfiguration générique " ${ }^{62}$ opérée par Ana María Matute dans sa réécriture sert ainsi à dire quelque chose sur son époque: lorsqu'on est adulte, le monde des fées n'existe plus. L'entrée dans le monde adulte, mais également dans le temps de l'histoire, est un désenchantement ${ }^{63}$.

\section{Dans le prolongement de Primera memoria: une double vérité}

El verdadero final de la Bella Durmiente n'est pas le premier texte dans lequel Ana María Matute expérimente avec le genre du conte, qu'elle détourne à des fins critiques. Elle a déjà utilisé certains contes et récits de littérature pour enfants de façon à dénoncer le régime franquiste tout en contournant la censure ${ }^{64}$, notamment dans sa trilogie de romans destinés aux adultes Los mercaderes ${ }^{65}$, et plus particulièrement dans Primera

6I. "Mais on doit supposer que, comme ces histoires ont l'habitude de terminer, ils furent tous très heureux. Bien que la Princesse ne serait jamais plus si naïve, ni le Prince si Charmant, ni les enfants si ignorants et sans défense.» (ibid., p. 81).

62. J'emprunte le concept de «(re)configuration générique» à Ute Heidmann : celuici permet «de comprendre l'inscription d'énoncés dans des systèmes de genres existants comme une tentative d'infléchir les conventions génériques en vigueur et de créer de nouvelles conventions génériques, mieux adaptées aux contextes socioculturels et discursifs qui changent d'une époque et d'une sphère culturelle et linguistique à l'autre" (U. Heidmann, J.-M. Adam, Textualité et intertextualité des contes, p. 34-35). Afin de sortir du paradigme qu'implique la notion de «genre» comme catégorie permettant de classer les textes, Ute Heidmann et Jean-Michel Adam ont développé les concepts de "généricité» et d' "effets de généricité», qui permettent "de penser la participation d'un texte à plusieurs genres" (J.-M. Adam, U. Heidmann, Le texte littéraire, p. 13).

63. Le désenchantement peut être considéré comme une caractéristique des réécritures de contes de fées du XXe siècle. A ce sujet, voir W. Mieder, Disenchantments.

64. A propos de l'usage des contes comme critique masquée, voir E. W. Harries, Twice Upon a Time.

65. Hormis Primera memoria, la trilogie est encore formée de Los soldados lloran de noche (1964) et de La Trampa (1969), dont est tirée la citation en exergue. 
memoria ${ }^{66}$, publié en 1960 . Ayant pour cadre le début de la guerre civile espagnole, ce roman met en scène la jeune Matia, qui doit aller vivre chez sa grand-mère, doña Praxedes, après que sa nourrice est tombée malade. Raconté à partir de la perspective de la jeune fille, le roman s'attache au passage de l'enfance à l'adolescence. Matia recourt aux classiques de la littérature enfantine, dont La sirenita et La reina de las nieves de Hans Christian Andersen, mais aussi Peter Pan de James Matthew Barrie, pour interpréter le monde adulte et les problèmes qu'elle rencontre. Dans ce texte, Matute "utilise les motifs du conte de fées pour représenter la perte de l'innocence qui caractérise le passage à l'âge adulte" ("uses fairy-tale motifs to chart the loss of innocence that characterizes the transition to adulthood $»)^{67}$. Les références aux lectures d'enfance permettent d'établir un contraste entre le présent vécu par Matia, celui de la guerre, et le temps de son enfance idéalisée, mais aussi de formuler une critique sociale et historique de ce présent. Comme je l'ai montré, c'est également le cas dans El verdadero final de la Bella Durmiente. Mais dans ce récit destiné à de jeunes lecteurs, la protagoniste ne considère plus les cuentos comme des métaphores de son enfance idéalisée: le merveilleux est constitutif de l'enfance, car la Bella Durmiente est désormais l'héroïne de son propre conte. Le contraste avec le monde réel est d'autant plus grand que la princesse a toujours vécu dans un univers peuplé de fées. Ana María Matute se montre ici particulièrement habile, car elle se sert de la première partie de La Belle au bois dormant comme $\mathrm{du}$ "pendant» enfantin de sa réécriture adulte. El verdadero final de la Bella Durmiente se place ainsi dans la continuité de ce que l'on peut appeler son projet poétique, établi de nombreuses années auparavant et traversant l'ensemble de son œuvre.

66. Primera memoria est le texte de Matute le plus étudié par la critique. Néanmoins, à ma connaissance, aucune étude n'a jamais été réalisée sur les relations qu'entretient ce texte avec El verdadero final de la Bella Durmiente. On notera que la grand-mère de Matia, doña Praxedes, et la reine ogresse Selva ont de nombreux points communs. De plus, la scène de la découverte de la tromperie par la reine Selva, qui trouve la Bella Durmiente et ses enfants cachés dans les combles de la maison de campagne, est en tout point identique avec une scène de Primera memoria, dans laquelle Matia et son cousin Borja se cachent de leur grand-mère dans la loggia. La scène de la découverte de la vérité est la plus importante de El verdadero final de la Bella Durmiente: ce n'est pas un hasard qu'Ana María Matute choisisse de faire retour à ce moment précis sur Primera memoria.

67. P. A. Odber de Baubeta, "The Fairy-Tale Intertext in Iberian and Latin American Women's Writing», p. 132, je traduis. 
María Elena Soliño note qu'il faut considérer les textes pour adultes d'Ana María Matute, et notamment Primera memoria, "comme des palimpsestes dont les structures supérieures reposent sur la base fournie par leur questionnement du genre du conte de fées» ("as palimpsests whose surface structures rest upon the base provided by their questioning of the fairy tale genre») ${ }^{68}$. De même, bien qu'il soit adressé aux enfants, El verdadero final de la Bella Durmiente peut être compris comme une réflexion sur ce que peut représenter un cuento aujourd'hui: comment, en d'autres termes, faire coexister le conte merveilleux avec les réalités crues et douloureuses de la dictature? La réécriture de Matute constitue une réponse possible, en marquant à la fois la continuité et la rupture d'avec le conte de fées. Par son titre et par les personnages qu'il met en scène, et par son éloignement du merveilleux au profit du fantastique, ce texte pousse le lecteur à s'interroger sur les rapports de la féerie avec la réalité. Anabel Sáiz Ripoll désigne ce texte comme un "conte de fées" ("cuento de hadas») ${ }^{69}$, mais je pense qu'il s'agit plutôt d'un "conte de non-fées» en cela que les fées ont disparu (mais non pas les sorcières et les ogresses). Marqué par la nostalgie d'une innocence perdue, il reflète une forme de désenchantement par rapport au temps présent, que $\mathrm{La}$ Belle au bois dormant de Perrault anticipe déjà dans la deuxième partie, sombre et cruelle, du récit. Le recours au fantastique et la disparition des fées permettent à Ana María Matute de poser la question de l'adéquation du genre merveilleux aux réalités inquiétantes du monde adulte, et plus spécifiquement d'aborder de façon indirecte l'histoire douloureuse de l'Espagne sous la dictature franquiste au $\mathrm{XX}^{\mathrm{e}}$ siècle. Sa réécriture met en scène le passage de l'enfance à l'âge adulte (sous-tendu par la guerre civile), et le passage du conte merveilleux au fantastique inquiétant, menaçant et déstabilisant. Cette réorientation du conte est significative, car comme Todorov l'a démontré, le fantastique permet d'articuler deux façons d'appréhender le monde. Matute explique pourquoi elle a récrit la fin de La Belle au bois dormant en ces termes:

68. M. E. Soliño, Women and Children First, p. 4, je traduis.

69. A. Sáiz Ripoll, "Ana María Matute, la mágica realidad», p. 14, je traduis. 
A mí me parece muy formativo, muy educativo que los nińos conozcan la verdad no sólo de la vida, sino también de la literatura. Debemos darnos cuenta de que la gran literatura es triste y es cruel, como lo es la vida ${ }^{70}$.

Le projet d'Ana María Matute est donc double. Le titre de sa réécriture peut se lire comme le désir de l'auteure de rappeler la fin trop souvent occultée du conte de Perrault, mais aussi de formuler une vérité sur la réalité de la guerre civile et du franquisme qui se dessine à travers sa réécriture du conte. En marquant une rupture définitive avec le monde de l'enfance, elle signale que la «vraie fin» est sans doute celle de l'innocence, ou de l'insouciance, qui s'endort à tout jamais. En ce sens, la malédiction réside peut-être moins dans le passage obligé à l'âge adulte que dans les circonstances particulières qui l'accompagnent. Tel est peutêtre le message de ce texte, qui témoigne de la cruauté du monde tel que vécu par Matute dans l'Espagne de Franco, où les fées guidant les héroïnes n'ont désormais plus leur place, et le happy end se réduit à une illusion ou à un mensonge d'Etat.

\author{
Sylvie Ravussin \\ CLE, Université de Lausanne
}

70. «Il me semble très formatif, très éducatif que les enfants connaissent la vérité non seulement de la vie, mais aussi de la littérature. Nous devons nous rendre compte que la grande littérature est triste et cruelle, comme l'est la vie.» (M.-L. Gazarian-Gautier, Ana Maria Matute, p. 44, je traduis). 


\section{BIBLIOGRAPHIE}

\section{Sources}

Matute, Ana María, Obra completa, t. IV, Barcelona, Ediciones Destino, 1975.

—, El verdadero final de la Bella Durmiente, Barcelona, Editorial Lumen, 1995.

Perrault, Charles, La Belle au bois dormant, in Histoires ou contes du temps passé. Avec des Moralitez, Paris, Claude Barbin, 1697, p. 1-46. Fac-similé reproduit in Contes, éd. par J. Barchilon, Genève, Slatkine, 1980.

\section{Travaux}

Adam, Jean-Michel, Lorda, Clara Ubaldina, Lingüistica de los textos narrativos, Barcelona, Ariel Lingüística, 1999.

Adam, Jean-Michel, Heidmann, Ute, Le texte littéraire. Pour une approche interdisciplinaire, Louvain-la-Neuve, Bruylant-Academia, 2009.

Gazarian-Gautier, Marie-Lise, Ana María Matute. La voz del silencio, Madrid, Editorial Espasa Calpe, 1997.

Harries, Elizabeth Wanning, Twice Upon a Time: Women Writers and the History of the Fairy Tale, Princeton, Princeton University Press, 2001.

Heidmann, Ute, Adam, Jean-Michel, Textualité et intertextualité des contes. Perrault, Apulée, La Fontaine, Lhéritier..., Paris, Classiques Garnier, 2010.

Hennard Dutheil de la Rochère, Martine, «But Marriage itself is no Party: Angela Carter's Translation of Charles Perrault's La Belle au bois dormant", in Marvels \& Tales, ed. by S. Benson, 
A. Treverson, Special issue on the Fairy Tale After Angela Carter, 24 (1) (2010), p. 131-151.

Jones, Margaret E. W., The Literary World of Ana Maria Matute, Lexington, The University Press of Kentucky, 1970.

Mieder, Wolfgang, Disenchantments. An Anthology of Modern Fairy Tale Poetry, Hanover/London, University Press of New England, 1985.

Odber de Baubeta, Patricia Anne, "The Fairy-Tale Intertext in Iberian and Latin American Women's Writing", in Fairy Tales and Feminism. New Approaches, ed. by Donald Haase, Detroit, Wayne State University Press, 2004, p. 129-147.

Pérez, Janet, "Contemporary Spanish Women Writers and the Feminine Neo-Gothic", Romance Quarterly, 51 (2004), p. 125140.

Prada, Juan Manuel de, «Yo no soy una erudita, ni falta me hace», $A B C$ literario, 16 de enero de 1998, p. 15-18.

Roas, David, De la maravilla al horror. Los inicios de lo fantástico en la cultura española (1750-1860), Vilagarcía de Arousa, Mirabel Editorial, 2006.

SÁrz Ripoll, Anabel, "Ana María Matute, la mágica realidad", Cuadernos de literatura infantil y juvenil, 84 (1996), p. 7-16.

Soliño, María Elena, Women and Children First: Spanish Women Writers and the Fairy Tale Tradition, Potomac, Scripta Humanistica, 2002.

Todorov, Tzvetan, Introduction à la littérature fantastique, Paris, Seuil, 1970.

\section{Crédit iconographique}

Fig. 1:

Dessin de Teresa Ramos, tiré de Matute, Ana Maria, El verdadero final de la Bella Durmiente, Barcelona, Editorial Lumen, 1995, p. 15. 
